\title{
Raman spectroscopy for label-free identification of calciphylaxis
}

William R. Lloyd

Shailesh Agarwal

Sagar U. Nigwekar

Karen Esmonde-White

Shawn Loder

Shawn Fagan

Jeremy Goverman

Bjorn R. Olsen

Dolrudee Jumlongras

Michael D. Morris

Benjamin Levi 


\section{Raman spectroscopy for label-free identification of calciphylaxis}

\author{
William R. Lloyd, ${ }^{a, \dagger}$ Shailesh Agarwal, ${ }^{b, \dagger}$ \\ Sagar U. Nigwekar, ${ }^{c}$ Karen Esmonde-White, ${ }^{d}$ \\ Shawn Loder, ${ }^{\mathrm{b}}$ Shawn Fagan,, Jeremy Goverman, \\ Bjorn R. Olsen, ${ }^{f}$ Dolrudee Jumlongras, ${ }^{f}$ \\ Michael D. Morris, ${ }^{\text {a,* }}$ and Benjamin Levi ${ }^{\text {,** }}$ \\ aUniversity of Michigan, Department of Chemistry, 930 North \\ University Avenue, Ann Arbor, Michigan 48109, United States \\ bUniversity of Michigan Medical School, Department of Surgery, 1500 \\ East Medical Center Drive, Ann Arbor, Michigan 48109, United States \\ cMassachusetts General Hospital, Department of Medicine, \\ 55 Fruit Street, Boston, Massachusetts 02114, United States \\ dUniversity of Michigan Medical School, Department of Internal \\ Medicine, 1500 East Medical Center Drive, Ann Arbor, \\ Michigan 48109, United States \\ eMassachusetts General Hospital, Department of Surgery, \\ 55 Fruit Street, Boston, Massachusetts 02114, United States \\ ${ }^{\mathrm{f}}$ Harvard University School of Dental Medicine, Department of \\ Developmental Biology, 188 Longwood Avenue, Boston, \\ Massachusetts 02115, United States
}

\begin{abstract}
Calciphylaxis is a painful, debilitating, and premorbid condition, which presents as calcified vasculature and soft tissues. Traditional diagnosis of calciphylaxis lesions requires an invasive biopsy, which is destructive, time consuming, and often leads to exacerbation of the condition and infection. Furthermore, it is difficult to find small calcifications within a large wound bed. To address this need, a noninvasive diagnostic tool may help clinicians identify ectopic calcified mineral and determine the disease margin. We propose Raman spectroscopy as a rapid, pointof-care, noninvasive, and label-free technology to detect calciphylaxis mineral. Debrided calciphylactic tissue was collected from six patients and assessed by microcomputed tomography (micro-CT). Micro-CT confirmed extensive deposits in three specimens, which were subsequently examined with Raman spectroscopy. Raman spectra confirmed that deposits were consistent with carbonated apatite, consistent with the literature. Raman spectroscopy shows potential as a noninvasive technique to detect calciphylaxis in a clinical environment. @ The Authors. Published by SPIE under a Creative Commons Attribution 3.0 Unported License. Distribution or reproduction of this work in whole or in part requires full attribution of the original publication, including its DOI. [DOI: 10.1117/1.JBO.20.8.080501]
\end{abstract}

Keywords: Raman spectroscopy; calciphylaxis, near-infrared light; light scatter; tissues.

Paper 150278LR received Apr. 28, 2015; accepted for publication Jul. 17, 2015; published online Aug. 11, 2015.

\footnotetext{
${ }^{*}$ Address all correspondence to: Michael D. Morris, E-mail: mdmorris@umich .edu; Benjamin Levi, E-mail: blevi@umich.edu

${ }^{\dagger}$ Authors contributed equally to this work.
}

\section{Introduction}

Calciphylaxis is a painful and debilitating condition affecting $1 \%$ to $4 \%$ of patients with end-stage renal disease or kidney transplants and its incidence is on the rise. ${ }^{1-4}$ Estimates of the 1 year survival rate and median survival time are about $46 \%$ and 1.5 years, respectively. ${ }^{5}$ Patients with calciphylaxis develop calcium deposits in their vasculature and soft tissues that may result in vessel thrombosis and tissue necrosis. Ultimately, patients develop large, open wounds that are painful and at high risk for infection. Current standard of care includes monitoring and managing pain, achieving hormone balance, and proper wound care to prevent potential secondary aggravations, such as infection and sepsis. ${ }^{6}$ Furthermore, the presence of co-morbidities, such as renal failure, may inhibit patients from undergoing multiple surgical interventions.

Since first reported in the literature in the 1960s and 1970s, calciphylaxis has been diagnosed by histological examination of suspicious tissues from at-risk patients (e.g., those with renal failure, dialysis, and elevated serum phosphate and/or calcium levels). ${ }^{6,7}$ However, histological examinations are destructive, time consuming, and require interpretation by a pathologist. Furthermore, debrided sites generally do not heal well, as blood flow to the region is limited due to calcified and thrombosed vessels and the lesion can actually increase in size. While radiographic techniques, such as computed tomography (CT), can detect calcium deposits, they are nonspecific for calciphylaxis, requiring a follow-up biopsy. Alternative diagnostic methods, such as analysis of serum solutes, have unclear utility. ${ }^{7}$ The advent of new treatment options for calciphylaxis patients, ${ }^{8}$ including pharmacologic agents (e.g., bisphosphonates, sodium thiosulfate, and cinacalcet $)^{9-12}$ and hyperbaric oxygen treatment, ${ }^{13}$ demands improved diagnosis.

A primary challenge in detecting calciphylaxis is finding small calcifications within a large wound bed. Previously, calcinosis has been examined in preliminary studies by infrared and Raman spectroscopy. ${ }^{14}$ A recent multimodal study of calciphylaxis tissue, ${ }^{15}$ including analysis via electron dispersive $\mathrm{x}$-ray analysis, confirmed calciphylaxis composition as carbonated apatite similar to bone mineral (as was hypothesized as early as the 1960s). ${ }^{16,17}$ To reliably detect this carbonated apatite, clinicians need a single noninvasive diagnostic instrument. Noninvasive diagnosis is important because any invasive diagnostic modality, such as debridement, can exacerbate the condition.

Furthermore, in the operating room, surgeons debriding calciphylaxis are not able to determine the complete extent of the disease visually and develop a treatment plan. Often this leads to excessive or inadequate debridement. Currently, hospitals and surgeons are reluctant to treat calciphylaxis for fear of exacerbating the condition. A diagnostic modality that determines the extent of the disease process would allow surgeons to confidently excise the margins of the calciphylactic tissue and assess the extent of the calcified lesions.

We propose rapid, noninvasive, and label-free Raman spectroscopy to improve the detection of calciphylaxis. Raman spectroscopy enables chemical fingerprinting of a sample with micron-level spatial resolution and subsurface probing properties similar to other tissue optical spectroscopies in the deep-red and near infrared regions. Raman spectroscopy has been previously employed in many investigations of calcified tissues, especially bone. ${ }^{18,19}$ These include characterizations of ex vivo bone biopsies ${ }^{20}$ in vivo ectopic bone formation, ${ }^{21}$ and human subject tibial plateaus. ${ }^{22,23}$ 

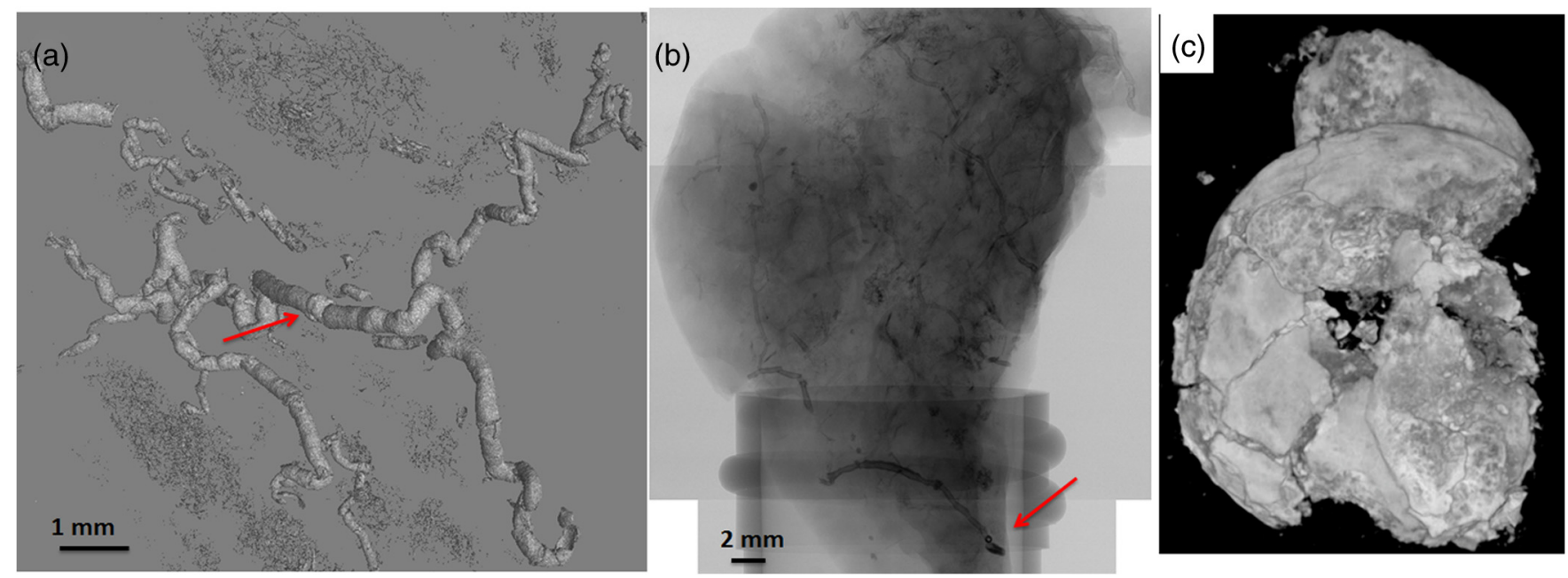

Fig. 1 Representative microcomputed tomography images from calciphylactic tissue. Arrows indicate location of measurements in ( $a$ and b) and (c) is close-up of nodule.

Here, we employ micro-CT and Raman spectroscopy to evaluate calciphylaxis biopsy specimens. The study was approved by the Institutional Review Boards at Massachusetts General Hospital and the University of Michigan. Six patients were enrolled. From each patient, a tissue biopsy was obtained, preserved in $3.7 \%$ formaldehyde and stored in a $70 \%$ ethanol prior to measurement. Micro-CT (Fig. 1 ; 5- to $12-\mu \mathrm{m}$ voxel size) confirmed the presence or absence of calcifications in each specimen. Of the six specimens, three specimens had extensive calcification, defined as nodules or regions in which blood vessels were externally calcified. These specimens were examined by Raman spectroscopy. Of the three other specimens, two had small, diffuse nodules embedded in fatty tissue, which is too thick for optical spectroscopy. Only one of these was examined by Raman spectroscopy. The other had no calcification detectable by micro-CT. Importantly, the presence of necrotic tissue did not always indicate calciphylactic mineral nodules.

Raman spectra were collected with either a Raman microprobe (interrogating a tissue area $\sim 0.4 \mathrm{~mm} \times 0.7 \mathrm{~mm}$ ) or a handheld fiber Raman probe (interrogating a tissue volume $<1 \mathrm{~mm}^{3}$ ). The Raman microprobe system was locally constructed ${ }^{24}$ (830 nm laser, $<20 \mathrm{~mW}$ laser power) and operated with 6 to $8 \mathrm{~cm}^{-1}$ resolution. The handheld fiber Raman probe measurements were conducted using a filtered $\mathrm{N}$-around-1 probe (EMVision, Loxahatchee, FL) connected to a portable Raman spectroscopy system (Rxn 1, Kaiser Optical Systems; 785-nm laser, $<20 \mathrm{~mW}$ laser power) operated with 6 to $8 \mathrm{~cm}^{-1}$ resolution. The Raman probe was fixed to a locally constructed stand to prevent motion artifacts.

For publication quality, the microprobe and fiber probe integration times were 120 and $60 \mathrm{~s}$, respectively. Probing the entire region of interest is currently limited by collection time. In principle, measurement time could be reduced to 1 to $3 \mathrm{~s}$. Spectra were preprocessed as previously described. ${ }^{25}$ The carbonated apatite phosphate band at $958 \mathrm{~cm}^{-1}$ was used as a signature of calciphylaxis.

Two specimens were examined with the microprobe and the third specimen was examined with the handheld fiber-optic probe. Microprobe measurements were guided by white-light imaging to identify regions of interest. Fiber probe measurements were guided by manual interrogation to identify hard regions suspected of containing calcified tissue. Then, Raman spectra were collected from suspicious tissue and nearby normal tissue. As needed, tissue specimens were sectioned to gain access to embedded calcifications. Both specimens examined with the Raman microprobe were classified as early stage by micro$\mathrm{CT}$, with calcified vasculature and calcium precipitate $<2 \mathrm{~mm}$ diameter. The specimen examined with the handheld fiberoptic probe was classified as late stage by micro-CT, with calcium precipitate nodules $>2 \mathrm{~mm}$ diameter. These specimens all yielded Raman spectra with phosphate Raman bands at $958 \mathrm{~cm}^{-1}$ (Fig. 2). As expected, spectra from nearby tissues lacked a band at $958 \mathrm{~cm}^{-1}$, indicating the absence of calcification. Raman microscopy of small diffuse nodules yielded spectra with strong lipid bands but no measurable phosphate bands.

The current study is limited by the small sample size. Because most debrided tissue is not calcified, most measurements were made on tissue that did not and should not generate a phosphate band. In the context of specimens from patients with calciphylaxis, when a tissue site yielded a strong apatitic phosphate signal, it originated from presence of calciphylaxis.

Our preliminary results suggest that Raman spectroscopy can be developed to improve clinical detection of calciphylaxis. Our previous experience is that spectroscopic evaluation of ex vivo specimens does not differ significantly from in vivo evaluation of superficial lesions. ${ }^{21}$

A key requirement for clinical translation is development of a handheld fiber-optic probe optimized to detect the clinical presentation of calciphylaxis. The probe must detect ectopic carbonated apatite at most locations, depths, and concentrations that occur in vivo. The probe must function despite varying tissue background, including contributions from serum, plaques, eschars, and lipids. Spectra must be collected in $<2 \mathrm{~s}$ and ideally, no more than $1 \mathrm{~s}$. With rapid measurements, clinicians would be able to map the wound area and characterize wound margins for treatment and debridement. Our early results do demonstrate that noninvasive tissue characterization using Raman spectroscopy can become a viable option for calciphylaxis diagnosis and evaluation of the extent of this debilitating process. 
(a)
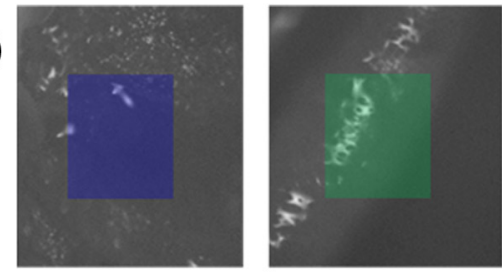

$958 \mathrm{~cm}^{-1}$,
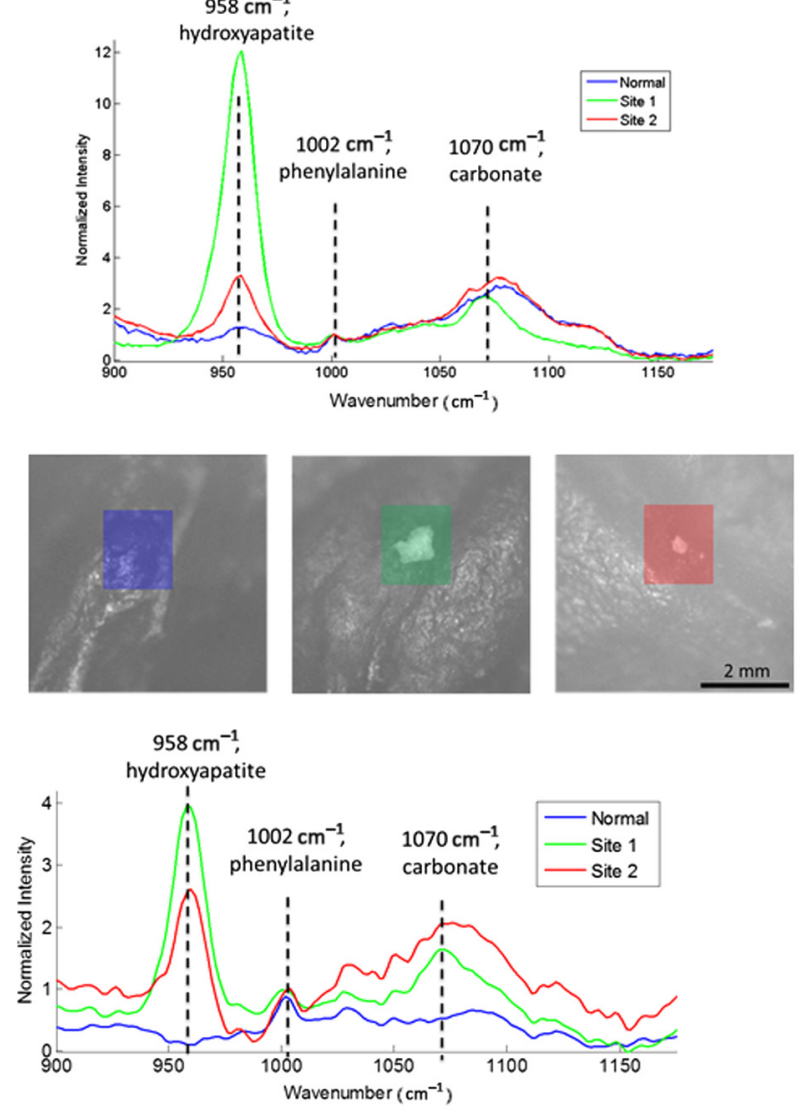

(b)

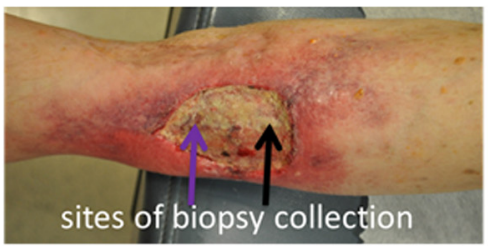

$958 \mathrm{~cm}^{-1}$,

hydroxyapatite

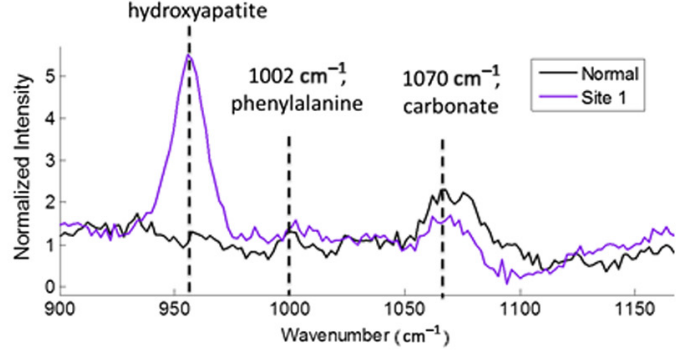

Fig. 2 Resected ex vivo tissue from patients presenting with calciphylaxis were examined by Raman spectroscopy. (a) Raman microprobe white-light imaging or (b) fiber-optic probe tactile response guided collocated Raman spectroscopy. Suspicious locations [green and red in (a), purple in (b)] had spectra consistent with apatitic mineral, noted by $958 \mathrm{~cm}^{-1}$ band.

\section{Acknowledgments}

Funding is gratefully acknowledged from NIH NIGMS K08GM109105-01, Plastic Surgery Foundation National Endowment Award, American Association of Plastic Surgery Academic Scholarship Award, DOD: W81XWH-14-DMRDP-CRMRPNMSIRA (to B. L.), a Pilot and Feasibility Grant from the Michigan Diabetes Research and Training Center, subgrant of NIH/NIDDK 2P30 DK020572 (K. E. W.), the NIH LRP, NIH F32, Coller Society, and the National Kidney Foundation and the American Heart Association (to S. U. N.), and NIH R01 AR052010 (to M. D. M.).

\section{References}

1. M. R. Hussein et al., "Calciphylaxis cutis: a case report and review of literature," Exp. Mol. Pathol. 86(2), 134-135 (2009).

2. L. R. Essary and M. R. Wick, "Cutaneous calciphylaxis. An underrecognized clinicopathologic entity," Am. J. Clin. Pathol. 113(2), 280287 (2000).

3. V. M. Brandenburg, M. Cozzolino, and M. Ketteler, "Calciphylaxis: a still unmet challenge," J. Nephrol. 24(2), 142-148 (2011).

4. S. Nigwekar et al., "Quantifying a rare disease in administrative data: the example of calciphylaxis," J. Gen. Intern. Med. 29(3), 724-731 (2014),

5. R. H. Weenig et al., "Calciphylaxis: natural history, risk factor analysis, and outcome," J. Am. A. Dermatol. 56(4), 569-579 (2007).

6. E. A. Ross, "Evolution of treatment strategies for calciphylaxis," Am. J. Nephrol. 34(5), 460-467 (2011).

7. A. R. Mazhar et al., "Risk factors and mortality associated with calciphylaxis in end-stage renal disease," Kidney Int. 60(1), 324-332 (2001).

8. C. Vedvyas, L. S. Winterfield, and R. A. Vleugels, "Calciphylaxis: a systematic review of existing and emerging therapies," J. Am. Acad. Dermatol. 67(6), e253-e260 (2012).

9. J. M. Beitz, "Calciphylaxis: an uncommon but potentially deadly form of skin necrosis," Am. J. Nurs. 104(7), 36-37 (2004).

10. S. Schliep, G. Schuler, and F. Kiesewetter, "Successful treatment of calciphylaxis with pamidronate," Eur. J. Dermatol. 18(5), 554-556 (2008).

11. H. Salmhofer et al., "Multi-modal treatment of calciphylaxis with sodium-thiosulfate, cinacalcet and sevelamer including long-term data," Kidney Blood Pressure Res. 37(4-5), 346-359 (2013).

12. S. U. Nigwekar et al., "Sodium thiosulfate therapy for calcific uremic arteriolopathy," Clin. J. Am. Soc. Nephrol. 8(7), 1162-1170 (2013).

13. J. An et al., "Hyperbaric oxygen in the treatment of calciphylaxis: a case series and literature review," Nephrology 20(7), 444-450 (2015).

14. S.-Y. Lin, "Biochemical and molecular aspects of spectral diagnosis in calcinosis cutis," Expert Rev. Mol. Med. 16, e6 (2014).

15. R. Kramann et al., "Novel insights into osteogenesis and matrix remodelling associated with calcific uraemic arteriolopathy," Nephrol. Dial. Transplant. 28(4), 856-868 (2013).

16. T. Coates et al., "Cutaneous necrosis from calcific uremic arteriolopathy," Am. J. Kidney Dis. 32(3), 384-391 (1998).

17. H. Selye, S. Grasso, and J. Dieudonne, "On the role of adjuvants in calciphylaxis," Rev. Allergy 15, 461 (1961).

18. M. D. Morris and G. S. Mandair, "Raman assessment of bone quality," Clin. Orthop. Relat. Res. 469(8), 2160-2169 (2011).

19. G. S. Mandair and M. D. Morris, "Contributions of Raman spectroscopy to the understanding of bone strength," BoneKEy Rep. 4, 620 (2015).

20. G. S. Mandair et al., "Potential of Raman spectroscopy for evaluation of bone quality in osteoporosis patients. results of a prospective study," Proc. SPIE 7548, 754846 (2010).

21. J. R. Peterson et al., "Early detection of burn induced heterotopic ossification using transcutaneous Raman spectroscopy," Bone 54(1), 28-34 (2013).

22. P. Matousek et al., "Noninvasive Raman spectroscopy of human tissue in vivo," Appl. Spectrosc. 60(7), 758-763 (2006).

23. K. Buckley et al., "Decomposition of in vivo spatially offset Raman spectroscopy data using multivariate analysis techniques," J. Raman Spectrosc. 45(2), 188-192 (2014).

24. J. D. P. McElderry et al., "Tracking circadian rhythms of bone mineral deposition in murine calvarial organ cultures," J. Bone Miner. Res. 28(8), 1846-1854 (2013).

25. F. W. L. Esmonde-White, K. A. Esmonde-White, and M. D. Morris, "Minor distortions with major consequences: correcting distortions in imaging spectrographs," Appl. Spectrosc. 65(1), 85-98 (2011). 